\title{
Aa. Vv., Cahiers Voltaire
}

\section{Olga Penke}

\section{OpenEdition}

\section{Journals}

\section{Édition électronique}

URL : https://journals.openedition.org/studifrancesi/39423

DOI : $10.4000 /$ studifrancesi.39423

ISSN : 2427-5856

\section{Éditeur}

Rosenberg \& Sellier

\section{Édition imprimée}

Date de publication : 1 décembre 2004

Pagination : $374-375$

ISSN : 0039-2944

\section{Référence électronique}

Olga Penke, «Aa. Vv., Cahiers Voltaire», Studi Francesi [En ligne], 143 (XLVIII | II) | 2004, mis en ligne le 30 novembre 2015, consulté le 19 mai 2021. URL : http://journals.openedition.org/studifrancesi/ 39423 ; DOI : https://doi.org/10.4000/studifrancesi.39423

Ce document a été généré automatiquement le 19 mai 2021.

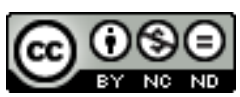

Studi Francesi è distribuita con Licenza Creative Commons Attribuzione - Non commerciale - Non opere derivate 4.0 Internazionale. 


\title{
Aa. Vv., Cahiers Voltaire
}

\author{
Olga Penke
}

\section{RÉFÉRENCE}

Cahiers Voltaire. Revue annuelle de la Société Voltaire, Ferney-Voltaire, publié avec le concours de la Fondation Voltaire à Ferney, $\mathrm{n}^{\circ} 1$ (2002) pp. 242.

1 La naissance d'une nouvelle revue spécialisée est un événement particulièrement réjouissant. Nous saluons chaleureusement la publication du premier numéro des Cahiers Voltaire et leur souhaitons une vie longue et fructueuse. Le comité de rédaction (Andrew Brown, Roland Desné, Ulla Kölving, André Magnan, Jean-Michel Raynaud) a conçu un projet prometteur et peu commun: faire connaître au lecteur l'état des recherches présentes et l'inviter également à participer aux débats et aux entretiens lancés par la revue. Les quatre parties permettent de réaliser cet objectif complexe: être en même temps savant, ouvert, pluriel et actuel. Le volume est aussi d'une beauté rare: une typographie bien choisie et des illustrations variées d'une qualité exceptionnelle procurent un plaisir supplémentaire.

2 Le premier ensemble, intitulé «Études et textes», fait montre d'éruditìon et d'esprit critique. L'étude d' André MAGNAN (Pour Marie-Louise Denis pp. 9-36) réfute une tradition biographique bornée à l'égard de la compagne de vie de Voltaire et reconstitue pas à pas l'histoire de leur relation, sous la lumière de lettres et d'autres documents. La publication par Ulla KöLVING de Deux lettres inédites d'Emilie Du Châtelet (pp. 75-82) démontre également que des précisions sont encore à souhaiter après la biographie critique fondamentale de René Pomeau. Des textes jusqu'ici inconnus de deux visiteurs de Voltaire montrent combien l'auteur a contibué lui-même à former son image, transformant son château en- centre intellectuel européen, et servant la diffusion de son œuvre et de sa pensée (Jean-Daniel CANDAUX, Alexandre Romanovitch Vorontsov raconte sa premiére rencontre avec Voltaire (1758) et François BESSIRE, Un après-midi chez Voltaire: récit inédit d'une visite à Ferney, pp. 105-107 et 109-113). Le but de l'étude de Jean-Noël PASCAL est d'attirer l'attention sur de nouveaux domaines de recherches ("Que la témérité 
de votre pied est grande»: quelques réflexions autour d'ÆEdipe travesti, parodie de la tragédie d'Edipe de M. de Voltaire, pp. 39-53). Cet article analyse le rapport entre Voltaire dramaturge et son public, ainsi que le rôle des parodies faites à partir de ses pièces. L'échantillon d'une version perdue du Siècle de Louis XIV et son commentaire est une contribution intéressante à l'image de Voltaire historien Andrew BROWN, Une version perdue du Siècle de Louis XIV, pp. 67-73). Les lettres du président Hénault sur le même texte, publiées la première fois par la présente revue, contenant beaucoup plus que de simples remarques ou d'appréciations, démontrent que deux conceptions de l'histoire et de la politique et deux manières d'écrire l'histoire s'y opposent. Derrière les critiques feutrées de Hénault se trouve une conviction traditionnaliste de l'histoire: «Le genre de l'Histoire, qui parle à la postérité demande un ton sérieux». Contrairement à lui, Voltaire ne refuse pas le débat (Ulla KöLVING-Andrew BRowN, Deux lettres inédites de Hénault à Voltaire sur le Siècle de Louis XIV, pp. 83-103). Deux articles sont consacrés à la réception ultérieure de Voltaire. Marc BUfFato (Voltaire selon Faguet, ou l'irréligion de Voltaire, pp. 55-65) montre comment les préjugés de Faguet concemant le XVII ${ }^{\mathrm{e}}$ siècle et son point de départ partial aboutissent à une image déformée. Une appréciation tout autre émerge de l'étude de Julien BENDA (Introduction au Dictionnaire philosophique de Voltaire, 1935, pp. 115-126) dont la réédition permet aux Cahiers de rendre hommage à l'auteur. Le "pacifisme mythique», c'est-à-dire la condamnation de toute guerre, est la grande leçon de Voltaire toujours actuelle selon Benda.

3 L'actualité des combats de Voltaire est évoquée par la partie «Débats» des Cahiers qui porte le sous-titre «Pour une archive des génocides». Ulla Kölving publie in extenso, avec des notes savantes et des variantes, un texte peu connu de Voltaire: Des conspirations contre les peuples, ou des proscriptions. Ce texte, destiné originellement à compléter la tragédie Triumvirat illustre l'histoire des génocides et la nécessité de la tolérance pour le lecteur contemporain (pp. 129-145). Une réflexion commune relève l'actualité de cette pensée de Voltaire de nos jours, tout en réalisant un dialogue entre les deux époques réflexives. La manipulation de tout ordre politique, la punition collective des peuples, les méfaits du fanatisme, les analogies possibles dans l'histoire sont mises en relief dans cette discussion animée et bouleversante (contributions écrites par Georges BENREKASSA, Francis BLANCHARD, Jérôme CARASSOU, Jean GOLDZINK, Stéphane HESSEL, Louis JANOVER, Roland MORTIER, Abdelaziz MOURDIE, Paolo QUINTILINI, Baldine SAINT-GIRON, Komla SOBO, Pierre tRUCHE et Piotr zBOROV, pp. 146-163). Bertram Eugène schWARZBACH ( $\mathrm{La}$ tentation de tuer le messager, ou l'évolution des valeurs morales, pp. 165-181) confronte ce même texte avec ses sources, pour mettre en relief les ressemblances et les procédés par lesqueis Voltaire a condensé la problématique présente dans maints ouvrages en une somme historico-philosophique. Il y défend Voltaire contre les accusations d'antisémitisme.

4 Le chapitre «Enquêtes» vise à réunir tous ceux qui s'intéressent à l'oeuvre de Voltaire dans un travail collectif continu, se servant de l'outil moderne et peu traditionnel: l'informatique. Ce premier numéro se penche sur deux questions. André Magnan coordonne une enquête sur les significations diverses du terme "voltairiens» qu'il propose de repérer dans l'histoire, dans le domaine français, mais également dans d'autres langues et d'autres cultures, auprès des lecteurs vivants hors de France (contributions d'André Magnan, Jacques Mény et Norbert Adeline, pp. 185-206). Françoise Tilkin propose de rassembler la documentation pour étudier au XVIII ${ }^{e}$ siècle «l'appartenance générique des contes» de Voltaire (pp. 207-212). 
5 Ce premier numéro particulièrement intéressant et riche se termine par la rubrique «Actualités», témoignant du caractère vivant des recherches sur Voltaire, qui seront certainement encore corroborées par cette revue. Les comptes rendus et une bibliographie internationale (livres, articles, thèses et mémoires) présentent les publications sur Voltaire parues en 2000-2001. 